\title{
ON A FIXED POINT THEOREM OF GREGUS
}

\author{
BRIAN FISHER \\ Department of Mathematics \\ University of Leicester \\ Leicester I.E1 TRH, England \\ and \\ SALVATORE SESSA \\ Istituto Matematico \\ Facolta' Di Architettura \\ Universita' Di Napoli \\ Via Monteoliveto 380134 Naples, Italy \\ (Received May 18, 1984)
}

ABSTRACT. We consider two selfmaps $T$ and $I$ of a closed convex subset $C$ of a

Barach space $x$ which are weakly commuting in $x$, i.e.

$\|T I x-I T x\| \leq\|I x-T X\|$ for any $x$ in $x$,

and satisfy the inequality

||$T x-T y|| \leq a|| I x-I y||+(1-a) \max \{|| T x-I x\|\| T y-,I y \|\}$

for all $x, y$ ir. $C$, where $0<a<1$. It is proved that if $I$ is linear and non-expansive in $C$ and such that IC contains $T C$, then $T$ and $I$ have a unique common fixed point in $C$.

KEY WORDS AHU PHRASES. Cormon fixed point, Banach space.

1980 MATHEMATICS SUBJECT CLASSIFICATION CODE. 54H25, $47 H 10$.

$\therefore \quad$ INTRODUCTION.

The second author [1], generalizing a result of Das and Naik [2], defined two mappings $T$ and $I$ of a metric space $(X, d)$ into itself to be weakly commuting if

$$
d(T I x, I T x) \leq d(I x, T x)
$$

for all $x$ in $x$. Two commuting mappings clearly satisfy (1.1) but the converse is not generally true as is shown with the following exanple:

EXAMPLE 1. Let $X=[0,1]$ with the Euclidean metric and aefine $T$ and $I$ by

$$
T x=\lambda /(x+4), I x=x / 2
$$

for all $x$ ir $x$. Then

$$
\begin{aligned}
d(\text { TIx, ITx }) & =\frac{x}{x+8}-\frac{x}{2 x+8}=\frac{x^{2}}{2(x+8)(x+4)} \\
& \leq \frac{x^{2}+2 x}{2(x+4)}=\frac{x}{2}-\frac{x}{x+4}=d(I x, T x)
\end{aligned}
$$

for all $x$ in $x$ but for any $x \neq 0$ :

$$
\text { TIX }=x /(x+8)>x /(2 x+8)=I T x .
$$


From now on, $c$ denotes a closed convex subset of a Banach space $x$. In a recent paper Gregus [3] proved the following theorem:

THEOREl: 1 . Let $T$ be a mapping of $C$ into itself satisfying the inequality

$$
\|T x-T y\| \leq a|| x-y\|+b\| T x-x\|+c|| T y-y\|
$$

for all $x, y$ in $c$, where $0<a<1, b \geq c, c \geq 0$ and $a+b+c=1$. Then $i$ has a unique fixed point.

Mappings satisfying inequality (1.2) with $a=1$ and $b=c=0$ are called nonexpansive and were ccinsidered by Kirk [4].

Wong [5] studied mappings satisfying inequality (1.2) with $a=0$ and $b=c=\frac{1}{2}$.

2. MAIN RESULTS.

We nov: prove the fo?lowing generalization of Theorem 1 :

THEOREM 2 . Let $T$ and $I$ be th'o weakly cormuting mappings of $C$ into itself satisfying the inequalizy

$$
\|T x-T y\| \leq a\|I x-I y\|+(1-a) \max \{\|T x-I x\|,\|T y-I y\|\}
$$

for all $x, y$ in $C$, where $0<a<1$. If $I$ is linear, nonexpansive in $C$ and such that IC contains $T C$, then $T$ and $I$ have a unique common fixed point in C.

PROOF. L.et $x=x_{0}$ be an arbitrary point in $C$ and choose points $x_{1}, x_{2}, x_{3}$ in $C$ such that

$$
I x_{1}=T x, I x_{2}=T x_{1}, I x_{3}=T x_{2} .
$$

This can te done since IC contains TC. Then for $r=1,2,3$ we have on using inequality (2.1)

$$
\begin{aligned}
\left\|T x_{r}-I x_{r}\right\| & =\left\|T x_{r}-T x_{r-1}\right\| \\
& \leq a\left\|I x_{r}-I x_{r-1}\right\|+(1-a) \max \left\{\left\|T x_{r}-I x_{r}\right\|,\left\|T x_{r-1}-I x_{r-1}\right\|:\right. \\
& =a\left\|T x_{r-1}-I x_{r-1}\right\|+(1-a) \max \left\{\left\|T x_{r}-I x_{r}\right\|,\left\|T x_{r-1}-I x_{r-1}\right\|\right\}
\end{aligned}
$$

and so

$$
\left\|T x_{r}-i x_{r}\right\| \leq\left\|T x_{r-1}-I x_{r-1}\right\| .
$$

It follows that

$$
\left\|T x_{r}-I x_{r}\right\| \leq\|T x-I x\|
$$

for $r=1,2,3$.

Further

$$
\begin{aligned}
\left\|T x_{2}-T x\right\| & \leq a\left\|I x_{2}-I x\right\|+(1-a) \max \left\{\left\|T x_{2}-I x_{2}\right\|,\|T x-I x\|\right\} \\
& \leq a\left(\left\|T x_{1}-I x_{1}\right\|+\|T x-I x\|\right)+(1-a)\|T x-I x\| \\
& \leq(1+a)\|T x-I x\|
\end{aligned}
$$


on using inequality (2.2). Thus

$$
|| \mathrm{Tx}_{2}-\mathrm{Ix}_{1}|| \leq(1+\mathrm{a})|| \mathrm{Tx}-\mathrm{Ix} \| \text {. }
$$

Ne will now define a point $z$ by

$$
z=\frac{1}{2} x_{2}+\frac{1}{2} x_{3}
$$

Since $C$ is convex the point $z$ is in $C$ and being $i$ linear, we have

$$
I z=\frac{i}{2} I x_{2}+\frac{1}{2} I x_{3}=\frac{1}{2} T x_{1}+\frac{1}{2} T x_{2} .
$$

It follows that

$$
\begin{aligned}
|| T z-I z_{1} \mid \leq & \frac{1}{2}\left\|T z-T x_{1}||+\frac{1}{2}|| T z-T x_{2}\right\| \\
\leq & \frac{1}{2}\left[a|| I z-I x_{1} \|+(I-a) \max \left\{\|T z-I z\|, \| T x_{1}-I x_{1}\right\}\right] \\
& \quad+\frac{1}{2}\left[a\left\|1<-I x_{2}\right\|+(1-a) \max \left\{\|T z-I z\|,\left\|T x_{2}-I x_{2}\right\|\right\}\right. \\
= & \frac{1}{2} a\left(\left\|I z-I x_{1}\right\|+|| I z-I x_{2} \|\right)+(I-a) \max \{\|T z-I z\|,\|T x-I x\|\}
\end{aligned}
$$

on using inequalities (2.1) and (2.2). Now

$$
\begin{aligned}
\left\|I z-I x_{1}\right\| & \leq \frac{1}{2}\left\|I x_{2}-I x_{1}\right\|+\frac{1}{2}\left\|I x_{3}-I x_{1}\right\| \\
& =\frac{1}{2}\left\|T i_{1}-I x_{1}\right\|+\frac{1}{2}\left\|T x_{2}-I x_{1}\right\| \\
& \leq\left(1+\frac{1}{2} a\right)|| T x-I x \|
\end{aligned}
$$

from inequalities (2.2) and (2.3) and

$$
\left\|I z-I x_{2}\right\|=\frac{1}{2}\left\|I x_{3}-I x_{2}\right\|=\frac{1}{2}\left\|T x_{2}-I x_{2}\right\| \leq \frac{1}{2}\|T x-I x\| .
$$

It follows that

$$
|| T z-I z|| \leq \frac{1}{4} a(3+a)\|T x-I x\|+(1-a) \max \{\|T z-I z\|,\|T x-I x\|\}
$$

and so

$$
\|T z-i z\| \leq \lambda .\|T x-I x\|
$$

where

$$
\lambda=\left(4-a+c^{2}\right) / 4<1 .
$$

We therefore have

$$
\inf \left\{|| T z-I z||: z=\frac{1}{2} x_{2}+\frac{1}{2} x_{3}\right\} \leq \lambda \text {.inf }\{|| T x-T x \|: x \varepsilon C\}
$$

and since we obviously have

$$
\inf \left\{\|T z-I z\|: z=\frac{1}{2} x_{2}+\frac{1}{2} x_{3}\right\} \geq \inf \{\|T x-I x\|: x \varepsilon C\} \text {, }
$$


it follows that

$$
\text { inf }\{\|T x-I X\|: x \varepsilon C\}=0 \text {. }
$$

Each of the sets

$$
K_{n}=\{x \in C:\|T x-1 x\| \leq 1 / n\}, H_{n}=\{x \in C:\|T x-I x\| \leq(a+1 j / a n\}
$$

( for $n=1,2, \ldots$ ) must therefore be non-empty and obviously

$$
k_{1} \supseteq k_{2} \supseteq \ldots \supseteq k_{n}
$$

Thus each of the sets $\overline{T K}$, where $\overline{T K_{n}}$ denotes the closure of $T_{n}$, must be nor-empty for $n=1,2, \ldots$ and

$$
\overline{\mathrm{TK}_{1}} \supseteq \overline{\mathrm{TK}_{2}} \supseteq \ldots \overline{\mathrm{TK}_{n}} \supseteq \ldots
$$

Further, for arbitrary $x, y$ in $k_{n}$,

$$
\begin{aligned}
\|T x-T y\| & \leq a\|I x-I y\|+(1-a) \max \{\|T x-I x\|,\|T y-I y\|\} \\
& \leq a(\|T x-I x\|+|| T x-T y\|+|| T y-I y\|)+(1-a) / n \\
& \leq(a+1) / n+a|| T x-T y \|
\end{aligned}
$$

and so

Thus

$$
|| T x-T y|| \leq \frac{a+1}{(1-a) n} \text {. }
$$

$\lim _{n \rightarrow \infty} \operatorname{diam}\left(T K_{n}\right)=\lim _{r_{1} \rightarrow \infty} \operatorname{diam}\left(\overline{T_{K}}\right)=0$.

It follows, by a well knowr result of Cantor (see, for example [6], p. 156), that the intersection $\bigcap_{n=1}^{\infty} \overline{T_{n}}$ contains exactly one foint $w$.

Now let $y$ be an arbitrary point in $\overline{T K_{n}}$. Then for arbitrary $\varepsilon>0$ there exists a foint $y^{\prime}$ in $k_{r}$ such that

||$T y^{\prime}-y||<\varepsilon$

and so, using the weak commutativity of $T$ and $I$ and the nonexpansiveness of $I$, we have irom (2.1) and (2.4):

$$
\begin{aligned}
\|T y-I y\| \leq & \left\|T y-T I y^{\prime}\right\|+\mid\left\|T I y^{\prime}-I T y^{\prime}\right\|+\left\|I T y^{\prime}-I y\right\| \\
\leq & a\left\|I y-I^{2} y^{\prime}\right\|+(1-a) \max \left\{\|T y-I y\|,\left\|T I y^{\prime}-I^{2} y^{\prime}\right\|\right\} \\
& +\left\|I y^{\prime}-T y^{\prime}\right\|+\left\|T y^{\prime}-y\right\| \\
\leq & a\left\|y-I y^{\prime}\right\|+(1-a) \max \left\{\|T y-I y\|,\left\|T I y^{\prime}-I T y^{\prime}\right\|+\left\|T y^{\prime}-I y^{\prime}\right\|\right. \\
& +1 / n+\varepsilon \\
\leq & a\left(\left\|y-T y^{\prime}\right\|+1 / n\right)+(1-a) \max \{\|T y-I y\|, 2 / n\}+1 / n+\varepsilon \\
\leq & (1+a) \varepsilon+(a+1) / n+(1-a) \max \{\|T y-I y\|, 2 / n\} .
\end{aligned}
$$

Since $\varepsilon$ is arbitrary it follows that

$\|T y-I y\| \leq(a+1) / n+(1-a) \max \{\|T y-I y\|, 2 / n\}$.

If $\|T y-I y\| \leq 2 / n$, then we have

$$
|| T y-I y|| \leq 2 / n<(a+1) / a n \text {. }
$$


If $\|$ Ty - Iy $\mid>2 / n,(2.5)$ implies

$$
\|T y-I y\| \leq(a+1) \cdot n+(1-a) \cdot\|T y-I y\| .
$$

So in bcth cases y lies in $H_{n}$. Thus $\overline{T^{\prime}} \subseteq H_{n}$ and so the point $w$ must be in $H_{n}$ for $n=1,2, \ldots$. It follows that

for $r_{1}=1,2, \ldots$ and so $T w=I w$.

$$
\| T w-I W \mid \leq(a+1) / a n
$$

Since (1.1) holds, we also have ITw = TIW. Thus

$$
\begin{aligned}
\left\|T^{2} w-T w\right\| & \leq a\|I T w-I w\|+(1-a) \max \left\{\left\|T^{2} w-I T w\right\|,\|T w-I w\|\right\} \\
& =a\left\|T^{2} w-T w\right\|
\end{aligned}
$$

and it follows that $T W=W^{\prime}$ is a fixed point of $T$ since $a<1$. Further $I W^{\prime}=I T W+T I W=T T W=T W^{\prime}=W^{\prime}$ and so $W^{\prime}$ is also a fixed point of $I$. Now suppose that $T$ and $I$ have a second common fixed pcint $w^{\prime \prime}$. Then

$$
\begin{aligned}
\left\|w^{\prime}-w^{\prime \prime}\right\| & =\left\|T w^{\prime}-T w^{\prime \prime}\right\| \\
& \leq a\left\|I w^{\prime}-I w^{\prime \prime}\right\|+(1-a) \max \left\{\left\|T w^{\prime}-I w^{\prime}\right\|,\left\|T w^{\prime \prime}-I w^{\prime \prime}\right\|\right\} \\
& \leq a\left\|w^{\prime}-w^{\prime \prime}\right\|
\end{aligned}
$$

and the unicueress of the common fixed point follows since $a<1$. This completes the proof of the theorem.

EXAIIPLE 2. Let $X=k$ and $C=[0,1]$ with the usual norm. Let $T$ and $I$ be as in example 1 . I is clearly linear and nonexpansive and further

$T C=[0,1 / 5] C[0,1 / 2]=I C$.

Thus

$\|T x-T y\|=\frac{4\|x-y\|}{(x+4)(y+4)} \leq \frac{1}{2} \cdot \frac{\|x-y\|}{2}=\frac{1}{2}\|I x-I y\|$

for all $x, y$ in $C$ and inequality (2.1) is satisfied for $a=1 / 2$.

So all the assumptions of Theorem 2 hold and $w=0$ is the unique common fixed point of $T$ and $I$.

Letting I be the identity mapping in Theorem 2, we have the following corollary which extends Theorem 1:

COROLI_ARY. Let $T$ be a nlapping of $C$ into itself satisfying the inequality

$$
\|T x-T y\| \leq a|| x-y \|+(1-a) \max \{\|T x-x\|,\|T y-y\|\}
$$

for all $x, y$ in $C$, where $0<a<1$. Then $T$ has a unique fixed point.

The result of this corollary was given in [7].

We note that the weak commutativity in Theorem 2 is a necessary condition. It suffices to consider the following example:

EXAMPLE 3. Let $X=R$ and let $C=[0,1]$ with the usual norm.

Define $T$ and $I$ by $T x=1 / 3, I x=x / 2$ for any $x$ in $C$. 
It is easily seen that all the conditions of Theorem 2 are satisfied except that of weak commutativity since with $x=1 / 2$

$$
\|T:(1 / 2)-I T(1 / 2)\|=1 / 6>1 / 12=\|T(1 / 2)-I(1 / 2)\| .
$$

However $T$ and $I$ do not have a common fixed point.

We conclude that although the mappings $T$ and $I$ in Theorem 2 have a unique common fixed point in $C$, it is possible for them to have other fixed points, as proved in the next example:

Example 4. Let $X=C=R^{2}$ with norm

$$
\|(x, y)\|=\max \{|x|,|y| \text { : }
$$

for all $(x, y)$ in $R^{2}$. Define mappings $T$ and $I$ on $R^{2}$ by

$$
T(x, y)=(0, y), I(x, y)=(x,-y)
$$

for all $(x, y)$ in $k^{2}$. Then for all $(x, y) \in R^{2},\left(x^{\prime}, y^{\prime}\right) \in R^{2}$

$$
\left\|T(x, y)-T\left(x^{\prime}, y^{\prime}\right)\right\|=\left|y-y^{\prime}\right|
$$

and

$$
\begin{aligned}
a !\left|I\left(x, y^{\prime}\right)-I\left(x^{\prime}, y^{\prime}\right)\right| \mid & +(1-a) \max \left\{|| T(x, y)-I(x, y)||,|| T\left(x^{\prime}, y^{\prime}\right)-I\left(x^{\prime}, y^{\prime}\right)||\right\} \\
& =a \max \left\{\left|x-x^{\prime}\right|,\left|y-y^{\prime}\right|\right\}+(1-a) \max \left\{|x|, 2|y|,\left|x^{\prime}\right|, 2\left|y^{\prime}\right|\right\} \\
& \geq a\left|y-y^{\prime}\right|+2(1-a) \max \left\{|y|,\left|y^{\prime}\right|\right\} \\
& \geq a\left|y-y^{\prime}\right|+(1-a)\left(|y|+\left|y^{\prime}\right|\right\} \\
& \geq\left|y-y^{\prime}\right|
\end{aligned}
$$

if $0<a<1$. Since $T$ commutes with $I$ and $I$ is a linear isometry, it follows that all the conditiors of Theorem 2 are satisfied but $T$ and $I$ each have an infinite number of fixec foints.

\section{REFERENCES}

1. SESSA, S. On a Heak Commutativity Condition of Nappings in Fixed Point Considerations, Publ. Inst. Math., 32 (46) (1982), 149-153.

2. DAS, K.M. and NAIK, K.V. Common Fixed Point Theorems for Commuting Maps on a Metric Space, Proc. Amer. Math. Soc., 77 (1979), 369-373.

3. GREGUS, Jr., M. A Fixed Point Theorem in Banach Space, Boll. Un. Mat. Ital., () $17-A(1980), 193-198$.

4. KIRK, W.A. A Fixed Point Theorem for Mappings Which do not Increase Distances, Amer. Math Monthly, 72(1965), 1004-1006.

5. WONG, Ch. S. On Kannan Maps, Proc. Amer. Math. Soc., 47 (1975), 105-111.

6. DUGUNDIJ, J. and GRANAS, A. Fixed Point Theory I, Polish Scientific Publishers, Warsawa (1982).

7. FISHER, B. Common Fixed Points on a Banach Space, Chung Juan J., XI (1982), 12-15. 


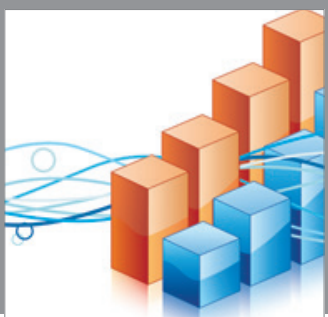

Advances in

Operations Research

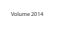

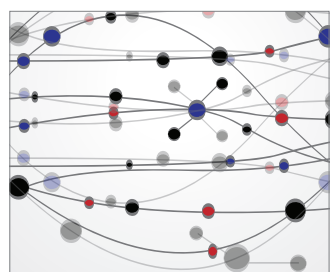

\section{The Scientific} World Journal
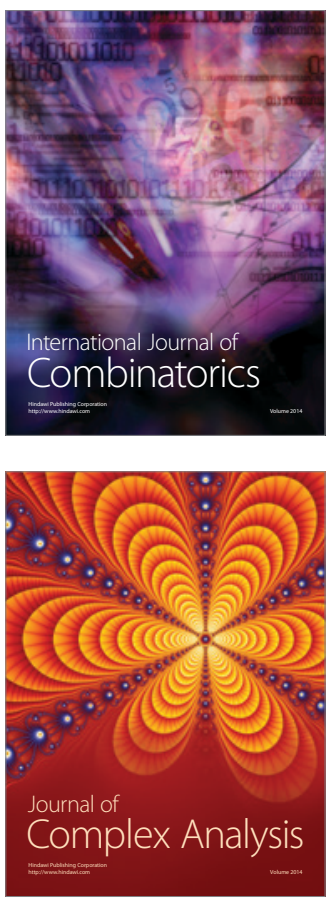

International Journal of

Mathematics and

Mathematical

Sciences
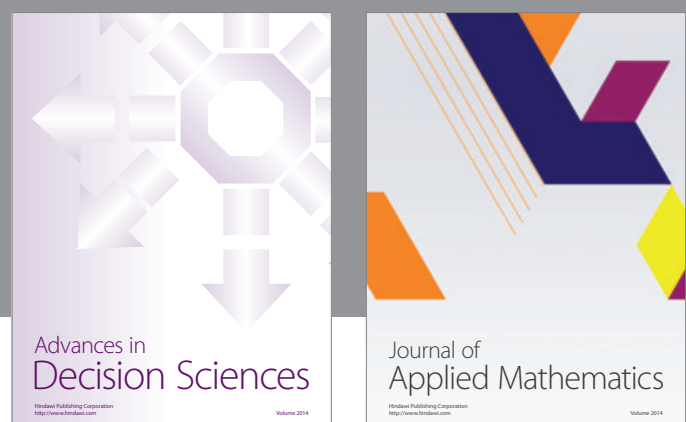

Journal of

Applied Mathematics
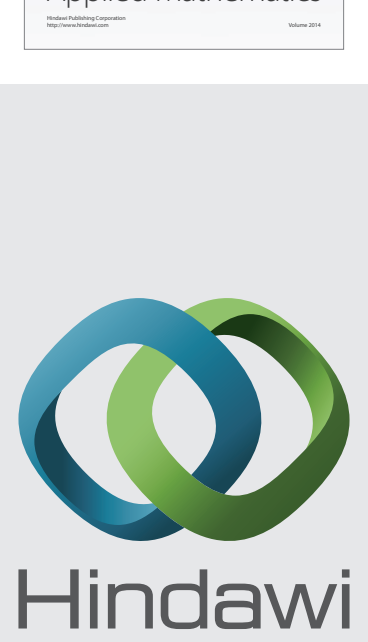

Submit your manuscripts at http://www.hindawi.com
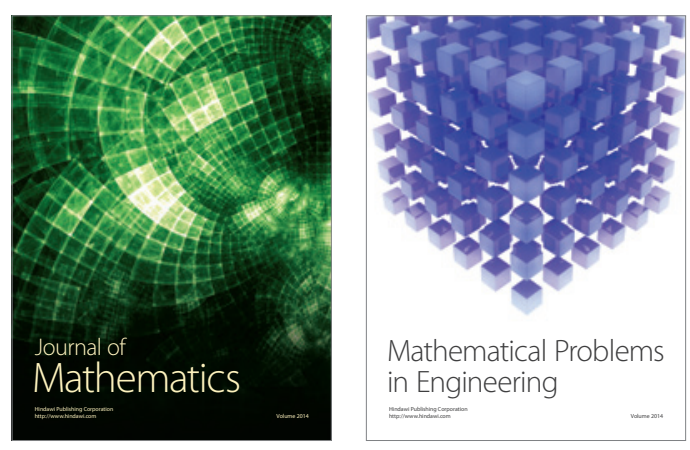

Mathematical Problems in Engineering
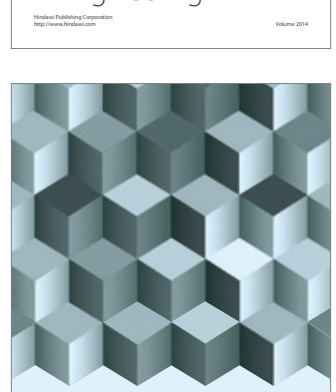

Journal of

Function Spaces
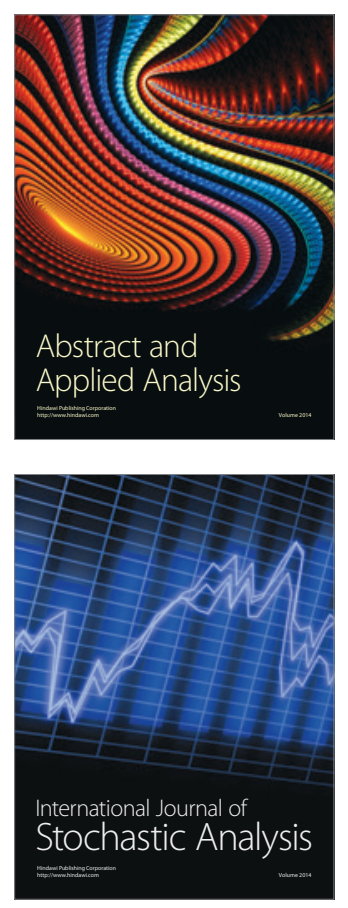

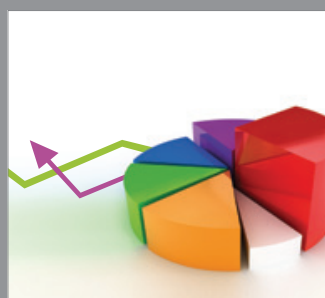

ournal of

Probability and Statistics

Promensencen
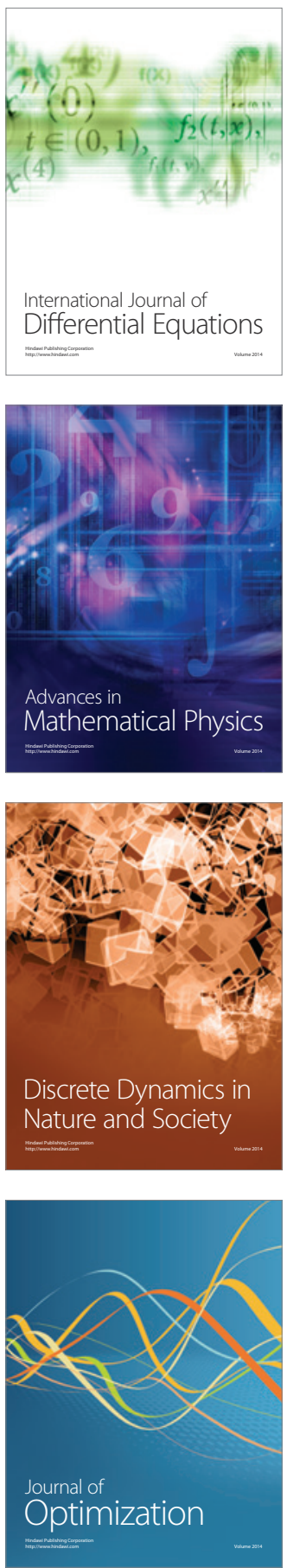